\title{
SUSANA SOCA: LA DAME À LA LICORNE
}

\author{
POR \\ FERNANDO LOUSTAUNAU \\ Asociación Uruguaya de Estudios Semióticos
}

\begin{abstract}
Tú la primera y la arbitraria... Ya apenas oigo tu voz esquiva, si no volviera, tu au sencia viva hasta la muerte, es necesaria. S.S.
\end{abstract}

No puedo comenzar este artículo sin hacer cómplice al lector de una situación que me resulta por demás inusual. Dicho de la manera más simple tal la paleta primaria del pintor-debo afirmar que la obra de Susana Soca no me gusta. Ahora bien, se trata entonces de la primera vez que abordo con efectivo interés un texto que no aparece bajo ese intuitivo deslumbramiento con que nos acercamos a nuestros objetos appetabilis. Se hace aún más particular esta instancia de aparente contradicción, cuando vuelvo a leer el prólogo de su primer libro, En un país de la memoria, y compruebo que la propia autora se preocupa en fundamentar ciertos reparos. Dice Susana Soca:

toda antologia implica una revisión que no puede hacerse sino en medio de dudas e inquietudes. Pero una antología de libros inéditos amenaza a su autor con una perplejidad que reclama dilucidaciones sin término.

El autor se encuentra en el estado de ánimo de alguien que hubiera quemado sus propios libros y perdido la memoria de los motivos que lo impulsaron a hacerlo, y luego, al azar, hallarse fragmentados en esos libros y sintiera la necesidad de verlos editados.

Ante todo predomina una sensasión de extrañeza; el autor se encuentra ante una obra arbitrariamente diferida, más que como autor como espectador de las aventuras externas y peripecias interiores que originaron el largo proceso. En mi caso particular, tengo la sensación de haber caminado sobre una cuerda tensa, sin abandonar ni editar. Ese tiempo y esa actitud, por la que el natural deseo de comunicación fuera fuertemente castigado, pesan sobre el orden actual de los poemas.

Un oficio lento y empecinado en el silencio, adquiere las caracteristicas del mundo en el cual se desenvuelve. Aquello que parece inherente a la producción poética, ciertas formas de comparación entre las propias cosas, la noción de su sentido general, toman el aspecto de una planta crecida en lo oscuro con relación a una planta necesitada de la luz. La obra se realiza en un secreto campo y otros climas le aparecen extranjeros .... 
A esta sucesión de sugerencias debemos incluir un hecho que no parece ser fortuito. Susana Soca murió sin ver sus libros publicados. Si bien tanto $E n$ un país de la memoria como su otro texto Noche cerrada habian sido ya preparados para una futura edición -hemos visto parte del prólogo del primero-, a su muerte ambos permanecian inéditos.

No hay necesidad de valerse de estas supuestas contradicciones para validar una intromisión en la vida de Susana Soca. Es claro que, de todos modos, hubiera sucumbido a la tentación de hurgar en el patrimonio interno de esta mujer solitaria. Pero es en cierto modo la propia autora quien nos avala en esta pretendida inserción biográfica: "Sin más historia que el poemal entro bruscamente en la historia mía".

A pesar que han transcurrido sólo treinta y dos años de la muerte de Susana Soca (acaecida el 11 de enero de 1959 en un accidente de aviación, en un viaje desde París con destino a Montevideo), muchos cambios se han producidoen su país. Casi todas las personas susceptibles de proporcionar signos relevantes de su existencia han desaparecido en los años posteriores a su muerte. Pero, sobre todo, se ha transformado de modo sustancial el modelo cultural en el que se formó Susana Soca. Una manifiesta traslación del imaginario colectivo se produjo en pocos años. Susana Soca resulta un paradigmático ejemplar de aquel Montevideo cuyo norte estético se fundaba y se observaba tomando como referencia orgánica a París. Una referencia que ni siquiera se veía necesario resaltar.

Muerte puntual, 1959, año en que el batllismo (fracción política que gobernara durante decenios, generadora de ese estado mesocrático, urbano, laico y civil que mereciera particular respeto) pierde por primera vez las elecciones nacionales, traspasando por tanto el poder al llamado Partido Nacional. Hecho que, interpretado con la sensibilidad uruguaya de entonces, representaba un remarcable acontecimiento histórico. $\mathrm{Y}$ si bien hoy no nos remite por cierto a transformaciones estructurales, puede bien ser tomado como involuntario símbolo -o voluntario por casualidad-de una serie de profundos cambios que se harán visualizables durante la década siguiente.

En la vida de Susana Soca, hubo un peculiar juego con el tiempo. Un destino - por llamarlo de algún modo- que la determinó a llegar tarde (o temprano) a sus espacios queridos. Cabe inferir que la vida no quiso darle el ejercicio del deseo. Asumió una ley tangencial, usufructuando de algún intersticio de la cultura dominante. En el número 12 de La Licorne - la revista que fundara en París en 1947 por "sugerencias" de su amigo Paul Eluard -nos relata su frustrado encuentro con Boris Pasternak. ${ }^{1}$ Para conocer en profundidad la obra

\footnotetext{
${ }^{1}$ En Sur 257, Buenos Aires (Marzo-Abril 1959), Guillermo de Torre escribe bajo el título

"Susana Soca, La Licorne y Pasternak":

Tratemos de hacer algunas conjeturas, libres desde luego pero perfectamente respetuosas, ante todo para la memoria de Susana Soca, y después para las precauciones o inhibiciones -no enteramente voluntarias - de Pasternak y sus amigos rusos. Porque hay un hecho que Susana
} 
del poeta ruso, Susana Soca aprendió el idioma. Luego se instaló en Moscú, pero nuncallegaron a conocerse. Puntual tardanza. Pasternak le escribe a Montevideo, pero ya es demasiado tarde. Suana Soca fue la primera en traducir al castellano poemas y prosas de Pasternak, cuando era cuasi un desconocido fuera de la Unión Soviética. Esas obras fueron publicadas en Montevideo cuando eran inéditas en su idioma original.

Los primeros años de Susana soca - desde esa hermenéutica autorizada que la historia llama historia - ya auguran un ser de características bien particulares. Hija única del reputado médico Francisco Soca -ya cincuentón a su nacimiento - y de una patricia montevideana, Luisa Blanco Acevedo descendiente de escritores, políticos y terratenientes-, la cual sobrevivirá a su hija diez años.

Nace Susana Soca en 1907, en medio de las transformaciones socializantes del presidente José Batlle y Ordóñez, las que buscarán apaciguamientos tranquilizadores para la clase dominante con su correligionario sucesor, Claudio Williman. En un Montevideo estratificado y cosmopolita (el censo de 1908 arrojó que el $41 \%$ de sus habitantes eran extranjeros, casi en su totalidad nacidos en Europa), con un creciente desarrollo económico que colocará al país en segundo lugar en el mundo en cuanto a reservas de oro per cápita y con leyes sociales progresistas presentadas en el Parlamento para escalofrío de los más conservadores. (La ley de divorcio, por ejemplo, ya era debatida en 1905, conociendo aprobación parcial pocos años después). Se perfilaba entonces esa dicha "Suiza de América" con que fue identificado el país; exportación batllista de fines no siempre fecundos pero suficientes, a nuestros efectos, para delinear una sociedad menos comprometida con la raigambre ibérica que las demás repúblicas del Continente.

Cuenta Susana sólo meses de vida cuando es llevada a París, ese epicentro compulsivo entonces para todo montevideano preciado. Yel matrimonio del Dr. Soca y Luisa Blanco Acevedo bien que lo era. Su bautizo ocurre en Notre Dame, primer signo, verdadero semeion, que la involucrará en la civilización francesa. En realidad su vida entera constituirá un tránsito. Una imagen solitaria que representa un movimiento continuo; una dynamis a la deriva, militante del silencio que no se cansó de absorber. De elaborar. Una mujer que parecía conocer desde un espacio anterior la escucha. Tal vez no sea apresurado aseverar que lo mejor de su obra quedó por ser escrito; que el tiempo de Susana Soca corría por diferentes cronómetros, no verificables en nuestra cultura posible. Susana no se autorizó ese desprendimiento último; accedió al estadio de una llamémosla

Soca no cuenta en su artículo pero que ella nos reveló a los amigos y que además ha trascendido por otros conductos. Y es que a punto de dejar Moscú, alguien entra a su habitiación del hotel e invocando el nombre de Pasternak, pone en sus manos un abul tado manuscrito con el encargo de traspasarlo a otras, una vez cruzada la frontera. El manuscrito era el del Doctor Zhivago y el destinatario definitivo resultó ser el editor Feltrinelli, de Turin". 
parábola gestual. Conocía el secreto a voces en que se refugia la cultura burguesa. En esa neurosis colectiva indispensable para el funcionamiento del sistema, eligió la liberación poética. Fue lentamente agotandolos divertimentos fungibles que ofrece la vida permitida, hasta su transplante en un lugar que no conoce retorno. Así quedó, divorciada de dos modelos que nunca terminó de abandonar. Su contacto, siempre tangencial, siempre esporádico, con corrientes estéticas fieles al inconsciente, la fueron transformando, obligándola a leerse, a releerse. Así, sus libros estaban "preparados", pero, a su vez, seguían esperando. Esperando ese desprendimiento que sólo podemos acotar en sus claves, un futur antérieur de una sucesión de desfasajes en el tiempo. Que podemos interpretar testamentarios: el legado es el puzzle de fechas, viajes, silencios, incompletudes y ese pasar revista implacable. Pasar (por la) revista, la otra mirada.

En su largo, tardío Fort da, cumple hasta el viaje último el recorridoinfantil y arcaico. Pero doblemente infantil -y perfecto de invertido- con el designio que enseña que los niños vienen de París. Esta niña de cigüeña desorientada, quiso en su curiosidad mayúscula verificar los mitos. Quedó atrapada en los meandros de las fronteras terribles de la poesía.

Así, con su espacio ritual subvertido, Susana Soca cumplió todos sus viajes a contrario sensu. En la primavera de 1947 aparece en París el primer número de La Licorne, bajo su dirección. Cumpliendo el anotado destino inverso, ya no será la vieja cultura francesa prodigando dadivosa sus vanguardismos al Uruguay, sino que será una uruguaya que in situ -en el ombligo entonces del conocimiento - difundirá figuras de los dos mundos. Una mujer dividida en pretensión de junción de sus patrimonios culturales. (Otras tres revistas fueron fundadas por uruguayos en Europa; Alfar (1923) de Julio Casal en La Coruña, Cercle et Carré (1930) de Joaquín Torres-García y Michel Suephor en París y Mundo Nuevo (1965) de Emir Rodríguez Monegal, también en París. Susana Soca colaboró en el período montevideano de Alfar en varias oportunidades. La primera en 1932, con un estudio de Rainer Maria Rilke y los Cuadernos de Malte Laurid Brigge.)

Otra huella de su tránsito tardío e inverso, lo brinda una segunda fecha clave. Si bien realiza numerosos viajes, Susana Soca no se radica de modo efectivo en París hasta 1938. Pocos meses después estalla la guerra, y se produce el exilio de muchos intelectuales franceses. Susana Soca se queda en París durante toda la ocupación nazi, residiendo en el célebre hotel Georges V. Hasta su compatriota y buen amigo Jules Supervielle se instala en Montevideo, donde permanecerá hasta el fin de la guerra. Susana Soca y su tránsito inverso: S.S. en el perfecto lugar equivocado.

En Aspectos de París escribe:

Viviamos en las tinieblas, sólo la luna maravillosamente disputaba a las tinieblas, por ella las calles estrechas e inimitables brillaban como piedras preciosas y los más nobles edificios dormidos sobre el río se sumergían en los reflejos del agua, con seducciones justificadas por la luz del día y que hacían 
pensar en un Debussy más profundo que el de los reflejos en el agua.

Era tal el silencio, que era verdaderamente el silencio del temor donde la presencia de lo terrible entraba y salía, como entraba y salía la muerte entre los silbidos esporádicos y donde el accidente por inculpado al azar y por sorpresa era más terrible todavía. Así la muerte en noviembre de 1943 entraba a las carreras de Auteuil.

Al mismo tiempo que partía el techo de un hospital donde en un cuarto un grupo de locas jugaban a las cartas ....

Estos años aparecen como sustanciales para conformar su situación de poeta. Yasí lo afirma ella misma, sintiéndose identificada con todos sus escritos a partir de 1940. Conoció por vez primera los apremios y la estrechez; algo que en otros términos ya bien conocía. Otro atraso por anticipación de una auscultadora de la mediocridad humana - esa estrechez del alma.

Susana Soca expresa conocer lo voluble de los espíritus apresurados, capaces sólo de ofrecer afectos envanescentes, pasajeros. Ella, en cambio, se satisfizo con el pasaje (comobillete, en la simbología del gesto-parábola aludido). Un salvocunducto hacia el silencio. Porque es a través del silencio que halla ese bálsamo creativo, sosegador de sus dos mundos. Su mundo arcaico, cultural, hecho a la medida de una cultura diseñada para servir a finalidades predeterminadas. Formación cultural que llamamos realidad. Un idealismo neurótico que funda en la religión y toda forma normativa, su apetencia de "concretud". Que hace del sujeto una metáfora provisional de un lenguaje general, aceptable. Pero, sobre todo, coercitivo. Y, por otrolado, la "irresponsable" energía que toda sociedad se toma el trabajo de perimir, desviar y orientar, catequizante. Susana Soca no había terminado de escrutar aún los mecanismos para ejecutar la fuerza del deseo. Estado “de pasaje”.

La respuesta fue un silencio militante. No fue capaz de generar una perversión activa del discurso, un materialismo semántico, una economía del lenguaje con potencial como para alterar su organización neurótica. Por ello, es sólo a través de signos desarticulados que podemos intentar traducir a esta heroína escurridiza. Porque Susana se introdujo en el idioma con suave inocencia femenina y terminó descubriendo la ficción en que nos confinamos para no vivir en el deseo. De ese descubrimiento - ese abandono parcial de la neurosis colectiva - no se recuperó más. La posibilidad de vivir su propio lenguaje, fue siempre ese estallido moméntaneo. Pasajera "de tránsito".

Su propio nombre, Susana, ${ }^{2}$ signo primero, oficia de registro. $S u(b) S a n a$ Soca Blanco, la hija del médico. Una para-normalidad que logra paliar instancia intermedia- la resolución entre la neurosis común y la perversión, entre la neurosis colectiva o lo que se llamará locura. Y ese estado de lactancia, es la marca del descubrimiento. Y sin que implique más que otro signo, me

${ }^{2}$ El nombre Susana, muy popular en la actualidad en el Uruguay, era poco frecuente en la generación de Susana Soca. 
autorizo una anécdota - con toda su aparente trivialidad - antes de que se pierda. Hubo un manifiesto interés de Henri Michaux por Susana Soca. Viajó incluso a Montevideo para hacer explícitos sus sentimientos, encontrando como respuesta por parte de Susana una aceptación condicionada a que no se encontraran sus cuerpos. De valernos del relato, cabría leer que Susana Soca hizo del descubrimiento su religión privada.

Soy el que duerme lejos sin figuras

soy el que apenas sueña que no sueña ...

Soy el que duerme lejos sin figuras

el que no mira y sin embargo ve ....

Susana Soca parece comprometida con el "estado de mito" que plantea Platón en el Fedro. Estado de poeta que se insinúa en Estatuas:
$\mathrm{Al}$ principio soñé que soñaría con algún ángel
Tarde, los sueños de los jóvenes vivos fueron mis sueños
$Y$ bruscamente me abandonaron
Luego soñé con los sueños salidos de las bocas de piedra de las estatuas ....

Aunque de modo parcial, Susana conoció el encantamiento del poeta. Entendió con mayúsculas y aceptó la servidumbre frente a las estatuas. Sabido es, las puertas de la comprensión analítica se cierran a partir de la comprensión.

La poesía fue referencia, continente a falta de interés en los juegos de las otras almas. Refugio último en un mundo contaminado de pérdidas, lleno de ausencias. Sentido de existencia para una mujer inacabada. Como la figura aún no esculpida. De ahí su veneración y su semejante resuelto, a esa obra terminada.

Asimismo encuentra de interés la incompletud amorosa en Sören Kierkegaard, acerca de quien escribe un breve ensayo. Dice:

En Culpable o no Culpable, vemos claramente cómo el asceta que crece dentro de él rechaza, por falta de verdadera religiosidad, el mismo mundo que el esteta no podía tolerar por su irritante falta de elegancia ...

Kierkegaard sufre su propia extrañeza, peroésta se le hace aún más visible cuando encuentra a Regina Olsen. Él mismo dice hasta qué punto cuánto había de bello y adorable en la juventud de esa criatura armoniosa y alegre que contrastaba con la propia tristeza. Y hasta qué punto la lúgubre disposición de ánimo a la que diera el místico nombre de acedía pesó sobre él, como si lo advirtiese por primera vez.

Después de un año de vacilaciones, desiste de su casamiento, provoca una difícil ruptura, y se dirige a Berlín, adonde vive con la mayor fantasía. Lógicamente la abandonada olvidó el abandono, pero el que abandonara no 
podía olvidar nunca nada. Había vivido de la ruptura durante el breve acuerdo y del acuerdo durante la larga ruptura. Cómicamente, el muy deseable olvido de la joven le causa la mayor indignación, y, trágicamente, el personaje de Regina sigue viviendo en el interior de Kierkegaard, y como en él todo deviene y se transforma, los actos del drama pasado se actualizan y renuevan mediante la idea fundamental kierkegaardiana de la repetición, que encontró mucho antes de Freud, y bajo cuya ley, infatigables formas viven y reviven con nuestra vida.

"Tódo me es inexplicable y más que nada yo mismo", dice nuestro autor. Y su historia sentimental permanece como una de las más extrañas del mundo. Por un lado, él mismo ha dicho que, al querer abrazar a la persona amada, solo encontraba angustiosamente una sombra. $Y$, por otro lado, que nunca logró la menor comunicación espiritual con ella ....

Esa misma expresión de la conocida "teoría de la repetición" también aparece en la propia obra creadora de Soca. Las pérdidas, la sucesión de pérdidas en una mujer que halla en la poesía la única tierra firme. Dice:

Me quitaron la antigua presencia que despacio me guardaba ...

Me quitaron la colina donde sólo se respira la flor sin nombre de flor que el juego de las distancias abre en el viento violento ...

Ya me he dado a las cosas que nunca fueron mías cesé de estar en algo antes de estar en todo No me quitaron lo que sumergido en $\mathrm{mI}$ recobro palmo a palmo y nuevamente me niegan y conceden las puertas de la noche...

Como si me moviera en el contrario punto de la más ancha rueda de estaciones ya presiento la sed de la rama inclinada ...

Me han cercenado de arboledas múltiples aclimatado a las distancias lúcidas y sin embargo busco a tientas a lo lejos sobre las rocas de una apartada colina en una noche que ni siquiera es la suya busco el color del mar, el color de nube que algún hilo de oro como el jaspe atraviesa.

En "Laberinto" Susana Soca aporta elementos de notoria transparencia. 
Aqui el poema largo interrumpido siempre

y varias veces terminado

poema escrito por una que yo no soy

Sé que la encuentro en la mitad,

sin final ni principio.

Pero ya no la busco

sólo busco el poema para empezar de nuevo ....

Apropósito de esta poesía, dice Esther de Cáceres (Introducción a la lectura de Susana Soca, Montevideo 1964):

El poema, que dice angustiado proceso de expresión y de vida, destaca sin embargo una seguridad absoluta: es la experiencia verdadera de quien canta y su relación leal con las cosas. Tan importante revelación de Laberinto es una de las claves de la experiencia poética de Susana Soca. Entre las angustiadas líneas del canto la clave resplandece....

Pero el signo más revelador de la presencia literaria de Susana Soca es su revista La Licorne. Desde su primera aparición en París de 1947, hasta su último número en Montevideo en 1961 (donde apareció desde 1953 con el mixturado nombre de Entregas de La Licorne), se caracterizó por el refinamiento formal y por el rigor en la selección de sus colaboradores. la revista alcanzó los 16 números, siempre coordinada por Soca, con excepción del último, precisamente de homenaje póstumo a la fundadora.

En el número 1 dice en su página primera: "Cahiers trimestriels de littérature publiés sous la direction de Susana Soca”. Agrega a continuación: "Textes réunis par Roger Caillois et Pierre David". La tirada es de 2,200 ejemplares. El diseño de la carátula corresponde a Valentine Hugo, quien seguirá luego haciéndolas en las ediciones sucesivas, incluidas las montevideanas.

En este primer número colaboran dos uruguayos: Jules Supervielle con "Genèse", poema escrito en el Océano Atlántico de retorno a Francia luego de terminada la guerra. Y Felisberto Hernández, en esos años viviendo en París y recibiendo la generosa amistad de Soca. Publica la primera traducción al francés de "El Balcón". También Jorge Luis Borges, con su conocido "Tlön, Uqbar, Orbis Tertius" escrito en 1940 en la ciudad uruguaya de Salto. Este texto, incluido originariamente en Antología de la literatura fantástica, cuenta con un post scriptum para La Licorne de tres páginas. En 1990 se conmemoró en Salto el aniversario con la presencia de Adolfo Bioy Casares en la que fuera residencia de Enrique Amorim, sitio adonde seguramente Borges escribió el texto.

Otras colaboraciones pertenecen a Maurice Blanchot ("L'Idylle"), Didier Desroches ("Je vis toujours"), Georges Shehadé ("S'il neige"), Pablo Neruda ("Hauteurs de Macchu-Picchu"), Octave Nadal ("Corneille et la notion du sublime”), T.S. Eliot (“La terre vaine”), Garcilaso de la Vega ("Réponse de l'Inca”), 
René Daumal (La mère-mot"), Charles-Albert Cingria ("Vair et Foudre"), Charmide ("La Baraque"), Georges Garampon ("Images des Anges") y Senancour ("Isabelle").

Por su parte, el último número de la revista, "Homenaje a Susana Soca", incluye el nombre de Guido Castillo "por la redacción". Colaboran los uruguayos Juana de Ibarbourou, Jules Supervielle, Alvaro Vasseur, Carlos Sabat Ercasty, Esther de Cáceres, Emilio Oribe, Ricardo Paseyro, Enrique Lentini y Guido Castillo. Los demás colaboradores serán E. M. Cioran con su "Elle n'était pas d'ici", José Bergamín, Jorge Luis Borges con su reconocido poema "Susana Soca”, Jorge Guillén, Giuseppe Ungaretti, Lanza del Vasto, María Zambrano, Henri Michaux, Sherban Sidéry y Marcel Jouhandeau.

Los colaboradores en el período montevideano de Entregas de La Licorne, fueron: en el primer número (1953) "Homenaje a Paul Éluard", Jean Cocteau, René Micha, José Bergamín, René Char, textos de Éluard y de Susana Soca. En el número 2(1953), Jorge Luis Borges, Rafael Alberti, José Bergamín, Francisco Romero, Fernando Pereda, Felisberto Hernández, Carlos Rodríguez Pintos, Carlos Real de Azúa, Silvina Ocampo, Julián Marías, Orfila Bardesio, Carlos Gurméndez(h), Eduardo Dieste, Ema Risso Platero, Ricardo Paseyro, Ángel Rama, Guido Castillo, J. Hellmut Freund, Jacobo Langsner, Sherban Sidéry y Antonio Larreta. En el número 3 (1954): "Homenaje a María Eugenia Vaz Ferreira": Alberto Zum Felde, M.B.A. Mendilaharsu, Esther de Cáceres, Sarah Bollo, Ángel Rama, Susana Soca y una antología de Vaz Ferreira. En el número 4(1954): Pierre Jean Jouve, José Bergamín, Henri Gouhier, Enrique Casaravilla Lemos, Adolfo Bioy Casares, Clara Silva, Herbert Steiner, Hugo von Hofmannsthal, Xavier Abril, Carlos Gurméndez(h), Ricardo Paseyro, Jacques Madaule, Guido Castillo. Con crónicas de Annie Barbaro de Teixeira, M. A. Couturier, Esther de Cáceres, Sherban Sidéry, Hans Platschek, Lorenzo Varela, José Bergamín y Susana Soca. En el número 5-6 (1955): Emil Cioran, Jorge Guillén, Juan David García Bacca, R. A. C. Du Vivier, Christopher Fry, María Zambrano, Dolf Sternberger, Ida Vitale, Felisberto Hernández, Guido Castillo, Carlos Real de Azúa, Ángel Rama y Susana Soca. Crónicas de Hellmut Freund, José Pedro Díaz, Luiz Campodónico, Arturo Sergio Visca, Manuel de Castro, Julio Casal, Ángel Rama y Susana Soca. En el número 7 (1956): Parte I, "Homenaje a Jules Supervielle": Esther de Cáceres, Felisberto Hernández, Carlos Rodríguez Pintos, Susana Soca y el propio Supervielle. En la Parte II: Karl Jaspers, Emilio Oribe, Jean Paulhan, Silvina Ocampo, José Suárez, Fernando Pereda, Grete Busch, y crónicas de Arturo Sergio Visca, André Razin, Hildegard Jacoby, y R. Botelho Gosálvez. En el número 8 (1956): Karl Jaspers, Karl L. Mayer, Thomas Mann, Dylan Thomas, Juan Carlos Onetti, Roberto Ibáñez, Juan García Bacca, Ricardo Paseyro, John Wain, Magdalena Harriague, Alberto Moravia, Enrique Lentini, Hans Busch, Julio Fernández y Juan Carlos Paz. Crónicas de J. R. Wilcock, Arturo Sergio Visca, Alberto Paganini, José Suárez, Antonio Larreta y Guido Castillo.

En el número 9-10 (1957): Boris Pasternak, Francis Ponge, Jorge Carrera 
Andrade, Rudolf Pannwitz, Theo Leger, J. R. Wilcock, Pier Paolo Pasolini, Fernando Pereda, Marcel Jouhandeau y Susana Soca. Crónicas de P. André Vincent, Harman Grisewood, José Aguerre, José Mora Guarnido y Guido Castillo. Notas de Arturo Sergio Visca, Guido Castillo y Domingo Bordoli. En el número 11 (1958): Carlos Vaz Ferreira, Anónimo: "The Cloud of Unknowing", Emilio Oribe, Martin Buber, José Bergamín, Dylan Thomas, Thomas Merton, Boris Pasternak, André Pieyre de Mandiargues, Bernard Collin, Enrique Lentini, Juan Carlos Onetti y Ema Risso Platero. Crónicas de Antonio Pagés Larraya, José Aguerre, y notas de Guido Castillo, Pablo Mañé Garzón y Alberto Paganini. Por último el número 12 (1959): Boris Pasternak, Henri Michaux, Martin Buber, Fernando Pereda, Elsa Morante, Julio C. da Rosa, Ana Franck, Ricardo Paseyro, Luc Dietrich y Susana Soca. Crónicas de Arturo Sergio Visca y Valentina Rosen. Notas de Guido Castillo y "A Susana Soca".

Esto constituye, en síntesis, todo cuanto podemos decir sobre Susana Soca en un artículo. Y más allá de toda epistemología (im)posible - en esta instancia informativa- es a partir de un relevamiento de signos de procedencia diversa que se puede accecer a la situación de esta mujer. Figura que la inercia colectiva uruguaya ha pretendido - ha logrado- desvertebrar de su aparato cultural. Susana Soca tal vez por fortuna no existe. Yen su silencio pertinaz, como en " $\mathrm{La}$ demente", sigue hablando sola.

La precisión de mis males se extiende a las cosas vagas por noches agotadoras he jugado con las máscaras $y$ he buscado la fatiga como si buscara el agua ni siquera alguna muerte acedía me llegaba ....

Entre mi sombra y yo misma crece tenebrosa planta doy vida a lo intolerable en mi visión prolongada ....

La demente canturrea dicen que no tengo nada ....

Repiten ella divaga yo digo que hay una línea por los puntos generada y hay un punto entre los puntos La demente ya no canta ni siquiera canturrea aunque aquí nadie descansa y es desconocido el sueño sueño que estoy transformada en alguien que apenas vive .... 


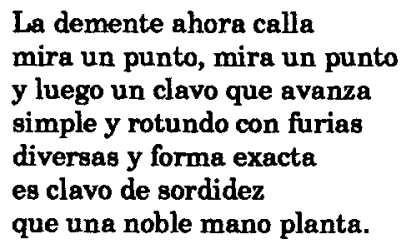

Susana Soca, entre otros logros, no recibe homenaje de índole alguna. Una escuela pública -la número 180 de Montevideo-lleva su nombre, pero por uno de esos malentendidos que abundan. A su padre en cambio, se le han prodigado honores múltiples. Hasta la localidad donde nacio, tiene hoy el nombre Soca. ${ }^{3}$

Susana Soca tal vez prefiera refugiarse en su Unicornio, del cual tan ricas lecturas se puede efectuar. Desde la alquimia que usa, hasta su ambivalencia para utilizarlo como símbolo de Monstrum Hermaphroditum. Símbolo de la castidad, aparece a su vez como emblemático de la espada o la palabra de Dios. Símbolo también de la sexualidad sublimada. En China, un animal casi semejante representa rectitud e ilustre nacimiento. Jung, en su obra sobre las relaciones de psicologia y alquimia, estudia diversos aspectos de este animal fabuloso; dice que se transmuta en paloma blanca.

Susana Soca Blanco, SANA SU CASO, CASO(LA SOCA) DE LOCA y su mito de origen. Diez letras perfectas de recorrido múltiple: Susana Soca y los tapices del Siglo XV del Museo Cluny. Supremacía "Ariana" en ciudad tomada. Tradición esotérica en cuerpo blanco. "Arbitrariamente diferida". Inédita.

s Una magnifica escultura, obra del reconocido Paul Bourdelle, homenajea al Doctor Francisco Soca en el Parque Batle de Montevideo; a cada costado, una figura femenina. Soca obtuvo su título de médico en Montevideo en 1883. "Bajo el padrinazgo del Prof. Charcot presenta en 1888 su tesis de doctorado en Paris sobre 'La enfermedad Friedreich'. En 1896 se le concede en la Facultad de Medicina de Montevideo la Cátedra de Clínica Médica, cargo que ocupará hasta su muerte en 1922. Formó una escuela médica impregnada de su formación francesa". Dr. A. Achard, "Influencias de la medicina de Francia en los médicos del Uruguay", El PaLs (1989). 
